\title{
Morbimortalidade por doenças oncohematológicas no Brasil entre 2002 e 2016
}

\author{
Morbidity and mortality due to hematologic neoplasms in Brazil between 2002 and 2016 \\ Morbilidad y mortalidade por neoplasias hematológicas en Brasil entre 2002 y 2016
}

Ramona Garcia Souza Dominguez ${ }^{1 *}$, Amanda Santos Veiga Freire ${ }^{1}$, Sabrina Souza Silva ${ }^{1}$, Natádina Alves Souza Campos ${ }^{1}$.

\begin{abstract}
RESUMO
Objetivo: Analisar a tendência temporal de morbimortalidade por doenças oncohematológicas no Brasil, entre 2002 e 2016. Métodos: Estudo descritivo de série temporal. Os dados foram extraídos dos sistemas nacionais de informação de Mortalidade e Internações Hospitalares, no período de 2002 a 2016, analisados no Excel e software STATA. Resultados: De modo geral, observou-se uma tendência crescente nas taxas de mortalidade e morbidade hospitalar por doenças oncohematológicas no Brasil entre 2002 e 2016. Para os óbitos, houve incremento anual de 0.04 óbitos/ano por Leucemias, de 0.03 /ano por Linfomas e de 0.04/ano por Mieloma, estatisticamente significante $(\mathrm{P}=0.000)$. O número total de homens falecidos por essas doenças foi superior ao de mulheres e as taxas de óbitos foram, para ambos os sexos, crescentes com a idade. As maiores taxas de hospitalização foram encontradas nos idosos, com maior proporção de atendimentos em caráter de urgência e diagnóstico principal de leucemias. As regiões Sul e Sudeste concentram quase $70 \%$ das mortes e das internações, porém houve um incremento importante nessas taxas no Nordeste. Conclusão: Os dados mostram o perfil das mortes e hospitalizações causadas por doenças oncohematológicas, sua distribuição regional e reafirmam a sua importância para a avaliação dos serviços de saúde em oncologia.
\end{abstract}

Palavras-chave: Mortalidade, Morbidade, Oncologia.

\begin{abstract}
Objective: To analyze the temporal trend of morbidity and mortality due to oncohematological diseases in Brazil, between 2002 and 2016. Methods: Descriptive study of time series. Data were extracted from the national information systems on Mortality and Hospital Admissions, from 2002 to 2016, analyzed in Excel and STATA software. Results: In general, there was an increasing trend in mortality and hospital morbidity rates due to oncohematological diseases in Brazil between 2002 and 2016. For deaths, there was an annual increase of 0.04 deaths/year by Leukemia, from 0.03/year per Lymphomas and 0.04/year for Myeloma, statistically significant $(P=0.000)$. The total number of men who died from these diseases was higher than women and the death rates for both sexes increased with age. The highest rates of hospitalization were found in the elderly, with a greater proportion of emergency care and a primary diagnosis of leukemia. The South and Southeast regions concentrated almost $70 \%$ of deaths and hospitalizations, but there was an important increase in these rates in the Northeast. Conclusion: The data show the profile of deaths and hospitalizations caused by oncohematological diseases, their regional distribution and reaffirm their importance for the evaluation of health services in oncology.
\end{abstract}

Keywords: Mortality, Morbidity, Medical oncology.

\footnotetext{
${ }^{1}$ Universidade Federal do Recôncavo da Bahia (UFRB), Santo Antônio de Jesus - BA.

*E-mail: ramonagarcia1@hotmail.com
} 


\section{RESUMEN}

Objetivo: Analizar la tendencia temporal de morbilidad y mortalidad por enfermedades oncohematológicas en Brasil, entre 2002 y 2016. Métodos: Estudio descriptivo de series de tiempo. Los datos se extrajeron de los sistemas nacionales de información sobre mortalidad y admisiones hospitalarias, de 2002 a 2016, analizados en el software Excel y STATA. Resultados: En general, hubo una tendencia creciente en las tasas de mortalidad y morbilidad hospitalaria debido a enfermedades oncohematológicas en Brasil entre 2002 y 2016. Para las muertes, hubo un aumento anual de 0.04 muertes/año por Leucemias, de 0.03 /año por

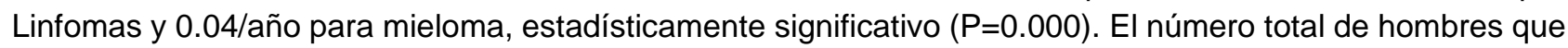
murieron por estas enfermedades fue mayor que el de las mujeres y las tasas de mortalidad para ambos sexos aumentaron con la edad. Las tasas más altas de hospitalización se encontraron en los ancianos, con una mayor proporción de atención de emergencia y un diagnóstico primario de leucemia. Las regiones sur y sudeste concentran casi el $70 \%$ de las muertes y hospitalizaciones, pero hubo un aumento importante en estas tasas en el noreste. Conclusión: Los datos muestran el perfil de muertes y hospitalizaciones causadas por enfermedades oncohematológicas, su distribución regional y reafirman su importancia para la evaluación de los servicios de salud en oncología.

Palabras clave: Mortalidad, Morbilidad, Oncología Médica.

\section{INTRODUÇÃO}

O câncer é um grave problema de saúde pública mundial e, no Brasil, foram estimados 625 mil casos novos para o biênio 2020-2022. Em 2018, dados do Globocan, divulgados pela Organização Mundial de Saúde (OMS), apontam a ocorrência de 33.519 casos novos de doenças oncohematológicas, 17.543 mortes e 90.512 pessoas vivendo com essas doenças no Brasil (5 anos após o diagnóstico) (FERLAY J, et al., 2018; INSTITUTO NACIONAL DE CÂNCER, 2019).

Leucemias, Linfoma não-Hodgkin (LNH), Linfoma Hodgkin (LH) e Mieloma Múltiplo (MM) afetam de diferentes formas o funcionamento da medula óssea e órgãos linfoides alterando a produção e função das células hematopoiéticas. A carcinogênese pode ser desencadeada pela exposição a fatores de risco como infecções por vírus, substâncias tóxicas e/ou causada por alterações genéticas herdadas, no entanto, grande parte dessas doenças não tem etiologia conhecida (HOWLANDER N, et al., 2017).

As manifestações clínicas das leucemias decorrem da falência medular e/ou infiltração de blastos nos tecidos, sendo diagnosticadas através de mielograma, exames de citogenética e imunofenotipagem. $O$ tratamento depende do tipo de leucemia, incluindo quimioterapia e transplante de células-tronco hematopoiéticas (TCTH).

Nos linfomas, podem ocorrer linfonodos ingurgitados, tosse, dispneia, dor torácica, febre, fadiga e perda ponderal, sendo diagnosticados por biópsia excisional do linfonodo ou tecido suspeito e o tratamento depende do tipo específico de LNH e LH, incluindo quimioterapia, radioterapia, imunoterapia e TCTH. No MM ocorre anemia, insuficiência renal, hipercalcemia e destruição óssea, sendo diagnosticado por exames laboratoriais, radiológicos e biópsia da medula óssea, ainda sem tratamento curativo (INSTITUTO NACIONAL DE CÂNCER, 2018; SALEMA CLZ e CARVALHO C, 2019).

As taxas de incidência das doenças oncohematológicas são mais altas nos países mais desenvolvidos, incluindo Austrália e Nova Zelândia, América do Norte e grande parte da Europa, mas as taxas de mortalidade são maiores nos países menos desenvolvidos (FERLAY J, et al., 2018). No Brasil, a distribuição dessas estimativas por sexo e região mostram que esses cânceres são mais incidentes nas regiões Sul e Sudeste, economicamente mais desenvolvidas (INSTITUTO NACIONAL DE CÂNCER, 2019).

Em todo o mundo são observadas diferentes tendências de incidência e mortalidade por doenças oncohematológicas. Nos Estados Unidos, para as Leucemias, a incidência tem aumentado em média 0,3\% ao ano, enquanto isso, as taxas de mortalidade apresentaram queda de 1,0\% ao ano no período de 2005 a 
2014. Para os Linfomas, observou-se taxas de incidência de 19.6/100 mil e 2.6/100 mil para Linfoma nãoHodgkin (LNH) e Linfoma de Hodgkin (LH), respectivamente, no período de 2013-2017. Enquanto isso, a mortalidade diminuiu em decorrência dos avanços terapêuticos na área, com taxas de 5.5/100 mil e 0.3/100 mil, respectivamente, para LNH e LH (FERLAY J, et al., 2018; HOWLADER N, et al., 2020).

$\mathrm{E}$, tanto no Brasil quanto no mundo, essas taxas são diretamente relacionadas às condições dos serviços de saúde e aos tratamentos disponibilizados, em concordância com a rapidez no diagnóstico. Ressalta-se nesse sentido, os entraves gerados pelo financiamento deficiente no Sistema Único de Saúde, os quais dificultam o alcance igualitário aos serviços de saúde no Brasil (GUERRA MR, et al., 2017; SILVA FF e LATORRE MRDO, 2020).

Considerando que os estudos sobre morbimortalidade por doenças oncohematológicas, especificamente em adultos, são escassos na literatura brasileira e, a fim de ampliar a discussão sobre a incidência dessas doenças na população, esse artigo agrega os dados de morbidade hospitalar comparando aos dados das mortes ocorridas no mesmo período.

A observação dessas tendências e o conhecimento sobre o perfil das internações e das mortes por essas doenças possibilitam avaliar a adoção de estratégias no âmbito das políticas públicas quanto à oferta de leitos hospitalares e/ou disponibilidade de tratamentos, considerando as diferenças regionais. Sendo assim, o objetivo deste estudo foi analisar a tendência temporal de morbimortalidade por doenças oncohematológicas no Brasil, entre 2002 e 2016.

\section{MÉTODOS}

Trata-se de um estudo descritivo, desenvolvido a partir da análise dos dados de óbitos e hospitalizações por doenças oncohematológicas no Brasil, no período de 2002 a 2016, nos indivíduos com 20 anos ou mais, disponíveis no Sistema de Informações sobre Mortalidade (SIM) e Sistema de Informações Hospitalares (SIH) do Ministério da Saúde (DATASUS/MS).

Foram considerados óbitos por doenças oncohematológicas, os registros codificados como causa básica, de acordo com a décima revisão da Classificação Estatística Internacional de Doenças e problemas relacionados à saúde (CID-10), com os códigos C81-C96 Neoplasias [tumores] malignas(os), declaradas ou presumidas como primárias, dos tecidos linfático, hematopoiético e tecidos correlatos. Para as hospitalizações foram considerados esses mesmos códigos para designar o diagnóstico principal da internação. Já as informações relativas aos dados demográficos da população brasileira no período de estudo foram obtidas do Instituto Brasileiro de Geografia e Estatística (IBGE).

Inicialmente, foram calculadas as taxas anuais brutas de notificação de óbito e hospitalização por doenças oncohematológicas por 100 mil habitantes, para cada região do país, segundo sexo e faixa etária. O crescimento anual das taxas foi estimado por modelos de regressão linear simples. Valores de $P<0,05$ foram consideradas estatisticamente significantes. Para o manejo de dados e análise foram utilizados os programas Excel e Stata, versão 12.

Por se tratar de um estudo com bases de dados secundários junto ao SIM e SIH, de acesso público com impossibilidade de identificação e contato com os sujeitos da pesquisa referidos pelos registros, de acordo com a Resolução no 510 do Conselho Nacional de Saúde, este estudo não foi submetido à apreciação de Comitê de Ética.

\section{RESULTADOS}

Nessa pesquisa, observou-se uma tendência crescente nas taxas de mortalidade por doenças oncohematológicas no Brasil entre 2002 e 2016. As taxas brutas de mortalidade apresentaram incremento anual de +0.04 óbitos/ano por Leucemias, de +0.03 /ano por Linfomas e de +0.04/ano por Mieloma, estatisticamente significante $(P=0.000)$. As taxas padronizadas de óbitos por 100 mil habitantes ao ano mostraram descenso de -0.07 mortes/ano por Leucemias e de $-0.06 /$ ano por Linfomas ( $P=0.000)$. Os resultados obtidos para Mieloma não foram significantes (Figura 1). 
Figura 1 - Mortalidade por doenças oncohematológicas. Brasil, 2002 a 2016

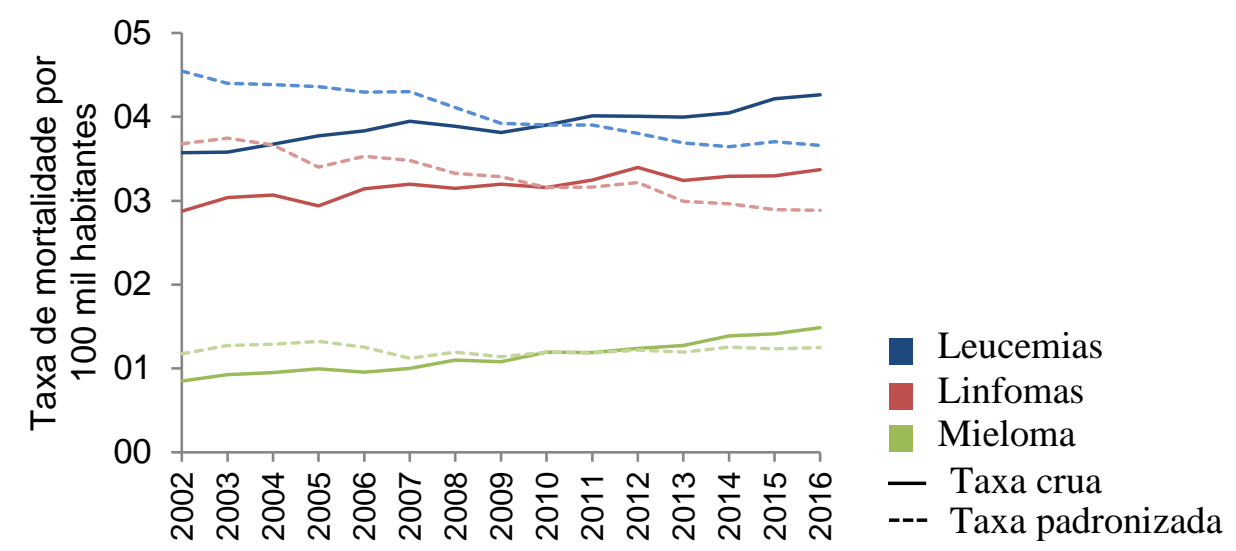

Fonte: Dominguez RGS, et al., 2020. Dados extraídos do SIM/DATASUS.

As taxas brutas de hospitalização por doenças oncohematológicas apresentaram tendência crescente com incremento anual de +0.07 internações/ano, estatisticamente significante $(P=0.000)$ (Figura 2).

Figura 2 - Hospitalização por doenças oncohematológicas. Brasil, 2002 a 2016.

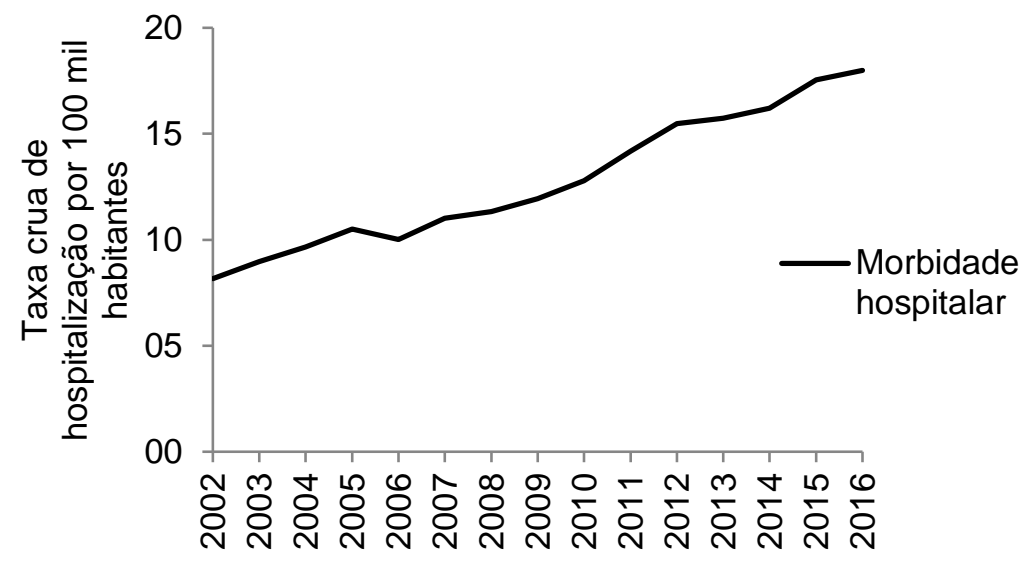

Fonte: Dominguez RGS, et al., 2020. Dados extraídos do SIH/DATASUS.

No período de estudo, ocorreram 369.819 internações hospitalares por doenças oncohematológicas, sendo que 42,3\% ocorreram por leucemias e 41,4\% por linfomas. Entre 2008 e 2016, a maioria das hospitalizações ocorreu em caráter de atendimento de urgência, com $14,2 \%$ de óbitos no desfecho desses atendimentos e maior proporção para as leucemias. Dos procedimentos clínicos, disponíveis no sistema de morbidade hospitalar e com código relacionado às doenças oncohematológicas, destacam-se os TCTH do tipo autogênico e intercorrências pós-transplante alogênico (Tabela 1). 
Tabela 1 - Caracterização das hospitalizações segundo aspectos clínicos. Brasil, 2008 a 2016.

\begin{tabular}{ccccccc}
\hline $\begin{array}{c}\text { Caráter do } \\
\text { Atendimento }\end{array}$ & \multicolumn{3}{c}{ Eletivo } & \multicolumn{3}{c}{ Urgência } \\
\hline Lista de Morbidade & $\mathbf{N}$ & $\%$ & Óbitos & $\mathbf{N}$ & $\%$ & Óbitos \\
\hline $\begin{array}{c}\text { Doença de Hodgkin } \\
\text { Linfoma não-Hodgkin }\end{array}$ & 9.216 & 11,0 & 323 & 13.993 & 7,8 & 1.289 \\
Leucemia & 30.125 & 36,0 & 2.237 & 58.621 & 32,6 & 7.591 \\
$\begin{array}{l}\text { Outras neoplasias } \\
\text { malignas - tecidos }\end{array}$ & 29.937 & 35,8 & 2.850 & 78.500 & 43,7 & 12.024 \\
linfoides hematopoiéticos \\
e relacionados
\end{tabular}

Fonte: Dominguez RGS, et al., 2020; Dados extraídos do SIH/DATASUS.

A comparação entre os anos de 2002 e 2016, demonstrou um aumento no total de óbitos e internações para ambos os sexos, para cada faixa etária e região. O número total de homens falecidos por doenças oncohematológicas foi superior ao de mulheres.

As taxas de óbitos foram, para ambos os sexos, crescentes com a idade. As maiores taxas de hospitalização foram encontradas nos idosos, principalmente, com 70-79 anos (61.4 por 100 mil) e a maior taxa de óbito na faixa etária de 80 anos ou mais (79.7 por 100 mil) (Tabela 2). 
Tabela 2 - Óbito e Hospitalização por doenças oncohematológicas segundo aspectos epidemiológicos. Brasil, 2002 e 2016.

\begin{tabular}{|c|c|c|c|c|c|c|c|c|c|c|c|c|}
\hline \multirow{3}{*}{$\begin{array}{r}\text { Ano } \\
\text { Variável }\end{array}$} & \multicolumn{6}{|c|}{2002} & \multicolumn{6}{|c|}{2016} \\
\hline & \multicolumn{3}{|c|}{ Óbito } & \multicolumn{3}{|c|}{ Hospitalização } & \multicolumn{3}{|c|}{ Óbito } & \multicolumn{3}{|c|}{ Hospitalização } \\
\hline & $\mathbf{N}$ & $\%$ & Taxa & $\mathbf{N}$ & $\%$ & Taxa & $\mathbf{N}$ & $\%$ & Taxa & $\mathbf{N}$ & $\%$ & Taxa \\
\hline \multicolumn{13}{|l|}{ Sexo } \\
\hline Masculino & 4.542 & 54,3 & 5.3 & 8.076 & 56,6 & 9.4 & 7.545 & 53,8 & 7.4 & 20.732 & 55,9 & 20.4 \\
\hline Feminino & 3.829 & 45,7 & 4.3 & 6.182 & 43,4 & 7.0 & 6.475 & 46,2 & 6.2 & 16.344 & 44,1 & 15.7 \\
\hline \multicolumn{13}{|l|}{ Idade } \\
\hline $20-29$ & 649 & 7,8 & 0.0 & 2.840 & 19,9 & 0.0 & 762 & 5,4 & 2.2 & 5.629 & 15,2 & 16.5 \\
\hline $30-39$ & 683 & 8,2 & 2.6 & 2.186 & 15,3 & 8.4 & 839 & 6,0 & 2.5 & 4.983 & 13,4 & 14.7 \\
\hline $40-49$ & 977 & 11,7 & 4.9 & 2.386 & 16,7 & 12.0 & 1.092 & 7,8 & 4.0 & 5.298 & 14,3 & 19.5 \\
\hline $50-59$ & 1.383 & 16,5 & 10.8 & 2.602 & 18,2 & 20.3 & 2.119 & 15,1 & 9.6 & 7.523 & 20,3 & 34.2 \\
\hline $60-69$ & 1.833 & 21,9 & 21.9 & 2.399 & 16,8 & 28.6 & 3.158 & 22,5 & 22.2 & 7.427 & 20,0 & 52.3 \\
\hline $70-79$ & 1.816 & 21,7 & 39.2 & 1.437 & 10,1 & 31.0 & 3.293 & 23,5 & 45.3 & 4.462 & 12,0 & 61.4 \\
\hline $80+$ & 1.030 & 12,3 & 55.0 & 408 & 2,9 & 21.8 & 2.757 & 19,7 & 79.7 & 1.754 & 4,7 & 50.7 \\
\hline \multicolumn{13}{|l|}{ Regiões } \\
\hline $\begin{array}{l}\text { Centro- } \\
\text { Oeste }\end{array}$ & 524 & 6,3 & 4.3 & 1.056 & 7,4 & 8.7 & 973 & 6,9 & 6.2 & 2.519 & 6,8 & 16.1 \\
\hline Nordeste & 1.059 & 12,7 & 2.2 & 1.752 & 12,3 & 3.6 & 2.268 & 16,2 & 4.0 & 7.511 & 20,3 & 13.2 \\
\hline Norte & 256 & 3,1 & 1.9 & 437 & 3,1 & 3.2 & 563 & 4,0 & 3.2 & 1.340 & 3,6 & 7.6 \\
\hline Sudeste & 4.575 & 54,7 & 6.1 & 8.152 & 57,2 & 11.0 & 6.899 & 49,2 & 8.0 & 17.810 & 48,0 & 20.6 \\
\hline Sul & 1.649 & 19,7 & 6.4 & 2.861 & 20,1 & 11.1 & 2.699 & 19,3 & 9.2 & 7.896 & 21,3 & 26.8 \\
\hline Total & 8.371 & - & 4.8 & 14.258 & - & 8.2 & 14.020 & - & 6.8 & 37.076 & - & 18.0 \\
\hline
\end{tabular}

Fonte: Dominguez RGS, et al., 2020. Dados extraídos do SIM/SIH/DATASUS.

As regiões Sul e Sudeste concentram quase $70 \%$ das mortes e das internações por doenças oncohematológicas no Brasil. As taxas de hospitalização foram menores na região Norte e nota-se um incremento importante nessas taxas, principalmente, para Nordeste (de 3.6 para 13.2) e Centro-Oeste (de 8.7 para 16.1) no período estudado.

A análise das taxas de mortalidade por doenças oncohematológicas, segundo sexo e idade, mostra que houve decréscimo anual entre as mulheres com 40 a 69 anos. Nos homens, houve aumento anual significativo apenas na faixa etária de 80 anos ou mais e tendência decrescente de 20 a 59 anos. Em relação às taxas de morbidade hospitalar, a mesma análise demonstrou tendências crescentes em todos os grupos etários para homens e mulheres, no período estudado (Tabela 3). 
Tabela 3 - Variação e análise de tendência da mortalidade e morbidade hospitalar por doenças oncohematológicas, segundo faixa etária e sexo. Brasil, 2002 a 2016.

\begin{tabular}{|c|c|c|c|c|}
\hline Sexo / Idade & Variação Anual & $\begin{array}{c}\text { Intervalo de confiança de } \\
95 \%\left(\mathrm{IC}_{95 \%}\right) \\
\end{array}$ & Valor de $\mathbf{P}$ & Tendência \\
\hline \multicolumn{5}{|c|}{ Óbitos Femininos } \\
\hline $20-29$ anos & $-0,01$ & $(-0,02 ; 0,01)$ & 0,220 & Estacionária \\
\hline $30-39$ anos & $-0,003$ & $(-0,02 ; 0,01)$ & 0,665 & Estacionária \\
\hline $40-49$ anos & $-0,60$ & $(-0,90 ; 0,28)$ & 0,001 & Decrescente \\
\hline $50-59$ anos & $-0,12$ & $(-0,20 ;-0,04)$ & 0,006 & Decrescente \\
\hline $60-69$ anos & $-0,10$ & $(-0,20 ;-0,01)$ & 0,035 & Decrescente \\
\hline $70-79$ anos & $-0,11$ & $(-0,41 ;-0,19)$ & 0,443 & Estacionária \\
\hline$\geq 80$ anos & 0,37 & $(-0,18 ; 0,92)$ & 0,166 & Estacionária \\
\hline \multicolumn{5}{|c|}{ Óbitos Masculinos } \\
\hline $20-29$ anos & $-0,02$ & $(-0,04 ;-0,004)$ & 0,022 & Decrescente \\
\hline $30-39$ anos & $-0,03$ & $(-0,05 ;-0,01)$ & 0,007 & Decrescente \\
\hline $40-49$ anos & $-0,10$ & $(-0,12 ;-0,08)$ & 0,000 & Decrescente \\
\hline $50-59$ anos & $-0,20$ & $(-0,26 ;-0,14)$ & 0,000 & Decrescente \\
\hline $60-69$ anos & $-0,13$ & $(-0,29 ; 0,02)$ & 0,089 & Estacionária \\
\hline $70-79$ anos & 0,25 & $(-0,04 ; 0,55)$ & 0,084 & Estacionária \\
\hline$\geq 80$ anos & 1,77 & $(0,96 ; 2,58)$ & 0,000 & Crescente \\
\hline \multicolumn{5}{|c|}{ Internações Hospitalares Femininas } \\
\hline $20-29$ anos & 0,36 & $(0,25 ; 0,47)$ & 0,000 & Crescente \\
\hline $30-39$ anos & 0,39 & $(0,32 ; 0,46)$ & 0,000 & Crescente \\
\hline $40-49$ anos & 0,35 & $(0,23 ; 0,47)$ & 0,000 & Crescente \\
\hline $50-59$ anos & 0,59 & $(0,35 ; 0,83)$ & 0,000 & Crescente \\
\hline $60-69$ anos & 1,29 & $(1,01 ; 1,56)$ & 0,000 & Crescente \\
\hline $70-79$ anos & 1,37 & $(1,02 ; 1,72)$ & 0,000 & Crescente \\
\hline$\geq 80$ anos & 1,13 & $(0,68 ; 1,57)$ & 0,000 & Crescente \\
\hline \multicolumn{5}{|c|}{ Internações Hospitalares Masculino } \\
\hline $20-29$ anos & 0,51 & $(0,39 ; 0,62)$ & 0,000 & Crescente \\
\hline $30-39$ anos & 0,36 & $(0,19 ; 0,52)$ & 0,000 & Crescente \\
\hline $40-49$ anos & 0,29 & $(0,12 ; 0,46)$ & 0,002 & Crescente \\
\hline $50-59$ anos & 0,51 & $(0,21 ; 0,80)$ & 0,003 & Crescente \\
\hline $60-69$ anos & 1,80 & $(1,42 ; 2,19)$ & 0,000 & Crescente \\
\hline $70-79$ anos & 2,63 & $(2,15 ; 3,12)$ & 0,000 & Crescente \\
\hline$\geq 80$ anos & 2,49 & $(2,0 ; 2,97)$ & 0,000 & Crescente \\
\hline
\end{tabular}

Fonte: Dominguez RGS, et al., 2020. Dados extraídos do SIM/SIH/DATASUS.

Também houve aumento crescente e significante $(P=0,000)$, das taxas de óbitos por doenças oncohematológicas para todas as regiões brasileiras. A região Sul obteve o maior incremento anual $(0.19$ óbitos por 100 mil habitantes), seguida pelo Nordeste (0.16/100 mil), Sudeste e Centro-Oeste (ambos com +0.13 óbitos $/ 100$ mil) e região Norte ( 0.10 óbitos por 100 mil habitantes ao ano).

As tendências também se mostraram crescentes para as taxas de hospitalização por doenças oncohematológicas $(p<0.001)$, para todas as regiões. $O$ crescimento anual foi mais acentuado no Sul $(+1.42 / 100$ mil) e Nordeste com +1.28 hospitalizações por 100 mil habitantes $(P=0.000)$, seguido do Sudeste $(+0.99 / 100$ mil), Norte $(+0.63 / 100$ mil) e Centro-Oeste $(+0.41 / 100$ mil) (Figura 3). 
Figura 3 - Mortalidade e Morbidade por doenças oncohematológicas segundo regiões. Brasil, 2002 a 2016.
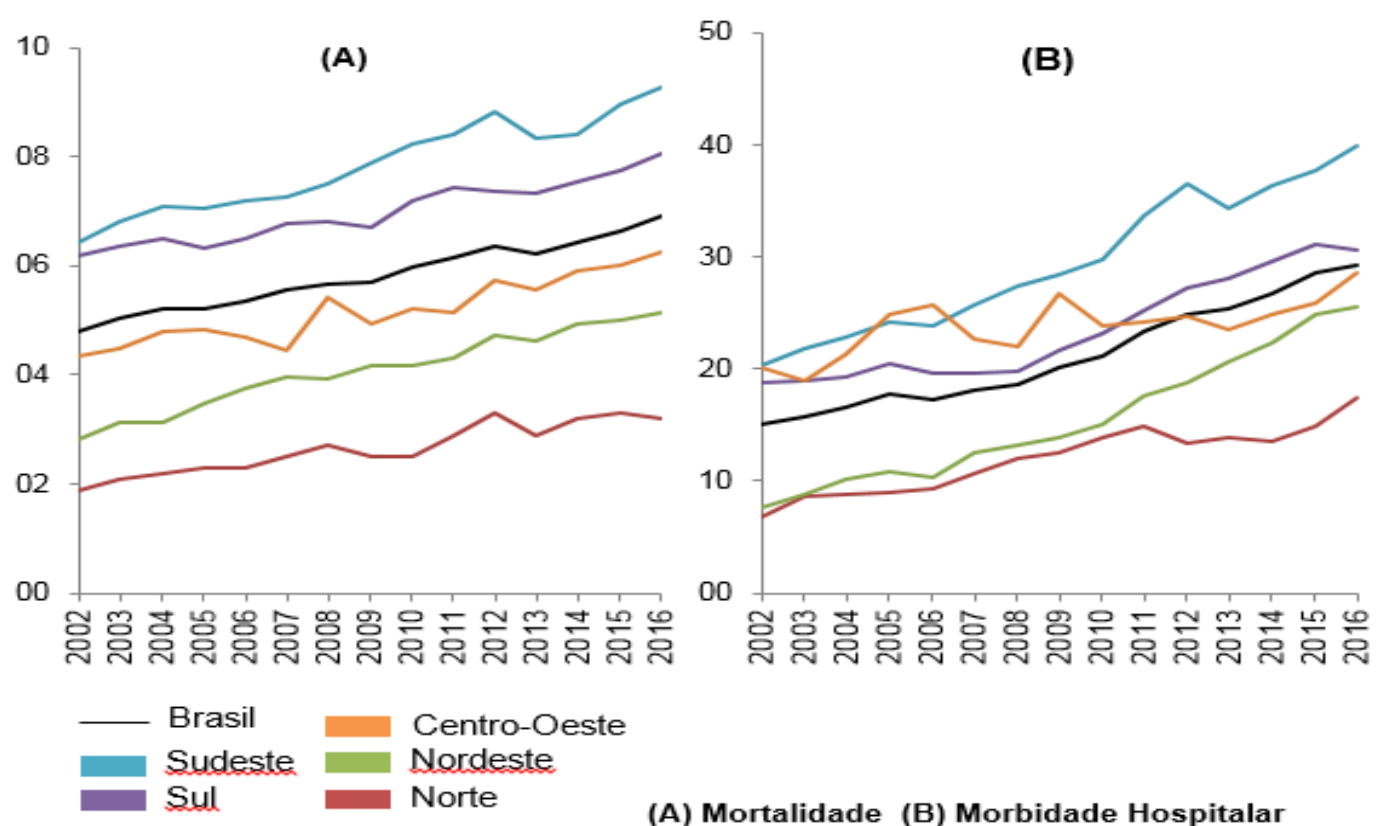

(A) Mortalidade (B) Morbidade Hospitalar

Fonte: Dominguez RGS, et al., 2020. Dados extraídos do SIM/SIH/DATASUS.

\section{DISCUSSÃO}

De 2002 a 2016, as taxas de mortalidade por leucemias, linfomas e mieloma em adultos apresentaram tendência crescente no Brasil, com diferenças entre os sexos e faixas etárias. De modo geral, morrem mais homens idosos. No Reino Unido, as taxas de mortalidade por doenças oncohematológicas também foram mais altas entre homens e nos idosos, principalmente, nas faixas etárias mais avançadas, no período de 2015-2017 (CANCER RESEARCH UK, 2020).

No período de estudo, a tendência de morbidade hospitalar manteve-se crescente em todas as faixas etárias, para ambos os sexos, porém, mais elevadas entre os homens e idosos, com maior frequência de leucemias $(42,3 \%)$ e linfomas $(41,4 \%)$. Resultados semelhantes foram demonstrados em um estudo brasileiro realizado a partir da análise de 590 prontuários em um Hospital Oncológico, entre 2004 e 2014. Em geral, os pacientes mais acometidos pelas neoplasias hematológicas tinham mais de 50 anos $(p<0,05)$, eram homens (56,8\%), provenientes de municípios do interior $(56,4 \%)$, não brancos e solteiros $(58 \%)$. As doenças mais prevalentes foram leucemias $(35,4 \%)$, linfomas $(26,8 \%)$ e mieloma $(15,8 \%)(p<0,05)$, sendo que $13,9 \%$ morreram durante a internação. Em relação ao tratamento, $91 \%$ (537) pacientes foram submetidos à quimioterapia e $8,4 \%$ (50) não fizeram uso de radioterapia e nem de quimioterapia (GRANJEIRO CF, et al., 2018).

O aumento nas taxas de mortalidade e morbidade hospitalar encontrados nessa pesquisa podem ser explicados por um conjunto de fatores: aumento na incidência por exposição aos fatores de risco conhecidos, incluindo a influência do envelhecimento populacional; melhorias no acesso ao diagnóstico e tratamento dessas doenças, que contribuíram para a definição do caso nos sistemas de mortalidade e morbidade; a influência dos tratamentos e outros aspectos associados à sobrevida dos doentes, principalmente, em faixas etárias mais avançadas, como demonstrado a seguir.

A padronização por idade das taxas de mortalidade por doenças oncohematológicas revelou estabilidade nos óbitos por Mieloma, com achatamento da curva de crescimento observada nas taxas cruas. Esse fato reafirma o envelhecimento populacional enquanto um dos fatores explicativos do achado de aumento no número de mortes por Mieloma, pois sabe-se que o risco de desenvolver a doença aumenta com a idade, com média de vida em torno de três a quatro anos, sendo que a taxa de sobrevida reduz drasticamente a partir dos 65 anos (SALEMA CLZ e CARVALHO C, 2019). 
Para as leucemias e linfomas, a comparação das taxas padronizadas e brutas revelou que o aumento da expectativa de vida no país e envelhecimento populacional explicam somente parte do aumento das taxas, pois, apesar da padronização, a tendência se manteve crescente no período, ou seja, existem outros fatores de risco associados com o aumento do número de casos e, consequentemente, das internações e óbitos.

Dentre eles, destacam-se o histórico familiar de neoplasias, etnia, doenças autoimunes, exposição a produtos derivados do petróleo, radiação ionizante e utilização de imunossupressores, além do sobrepeso e obesidade (HAGSTRÖM H, et al., 2020). Deve-se considerar ainda outros fatores intervenientes como a qualidade dos sistemas de informação e as mudanças no diagnóstico e no tratamento dessas doenças em cada Estado e regiões brasileiras (BOCCOLINI PMM, et al., 2015).

Neste estudo, os dados demonstraram que homens idosos tiveram mais internações hospitalares e morreram mais por doenças oncohematológicas do que as mulheres. O mesmo perfil foi encontrado em um Serviço de Oncologia que identificou $60,9 \%$ ( $n=81 / 133)$ de indivíduos do sexo masculino e $54,9 \%$ de idosos com 60 anos ou mais ( $n=73 / 133$ ) diagnosticados com doenças oncohematológicas, no período de 2014 a 2018. As maiores taxas de diagnóstico foram observadas nos anos em que houve a implantação da especialidade de Hematologia no município estudado e 10,5\% dos casos não foram submetidos a nenhum tratamento devido à idade ou doença avançada (OLIVEIRA MD, et al., 2020).

A expansão da rede de atenção oncológica contribuiu para o aumento da capacidade e do acesso ao diagnóstico de cânceres, o que também explica o aumento nas taxas de mortalidade e morbidade hospitalar. $\mathrm{Na}$ alta complexidade, a Política Nacional de Atenção Oncológica estabelece a garantia do acesso aos serviços para estadiamento e tratamento dos cânceres por meio da implantação de Unidades de Assistência de Alta Complexidade em Oncologia (UNACON) e Centros de Assistência de Alta Complexidade em Oncologia (CACON) (BRASIL, 2005).

Ainda que possa existir um longo caminho entre a política concebida e a implementação dos serviços, no período de estudo houve o incentivo para a habilitação de estabelecimentos de alta complexidade em Oncologia no Brasil. Em 2008, existiam 237 estabelecimentos de saúde habilitados na atenção de alta complexidade em oncologia apresentando um aumento progressivo nos anos seguintes, ainda que de forma desigual entre os Estados. Em 2019, atingiu-se o número de 351 estabelecimentos de saúde com habilitação para diagnosticar e tratar pacientes oncológicos (BRASIL, 2019).

Em 2014, foram investidos mais de 195 bilhões de dólares americanos nos serviços de saúde brasileiros, passando de um gasto per capita de $\bigcup \$ 201,00$ no ano de 2002 para $\bigcup \$ 947,00$ em 2014. No entanto, a análise mais detalhada revela que o aumento dos gastos com o financiamento do sistema de saúde não significa que as condições de saúde da população melhoraram, sendo necessária a gestão adequada dos recursos obtidos, pois o investimento no sistema de saúde privado supera os valores gastos com a saúde pública (OMS, 2020; SALDIVA PHN e VERAS M, 2018).

Assim, o investimento realizado no serviço público de saúde mostrou-se insuficiente para reduzir as taxas de mortalidade por doenças oncohematológicas que continuaram crescentes de 2002 a 2016. Ainda que tenham ocorrido melhorias no diagnóstico dessas doenças, o acesso à rede de atenção oncológica ainda não é tempestivo e equitativo, o que contribui para o diagnóstico tardio e redução da sobrevida (BRASIL, 2011).

De fato, as diferenças de mortalidade por doenças oncohematológicas segundo a faixa etária podem estar associadas ao diagnóstico tardio, pois esse interfere na proposta terapêutica. Como para outros tipos de cânceres, o sucesso do tratamento está relacionado com a precocidade da identificação da doença (SALEMA CLZ e CARVALHO C, 2019).

A análise dessas taxas de óbito segundo sexo e faixa etária mostrou tendência decrescente em mulheres com 40 a 69 anos e homens de 20 a 59 anos. As melhorias nos tratamentos das doenças oncohematológicas também podem explicar esses achados, assim como ocorreu em outros países, particularmente, quando observa-se a tendência crescente na morbidade hospitalar, com mais de $60 \%$ dessas internações nos grupos de 20-59 anos, para ambos os sexos (SANT M, et al., 2015; YE X, et al., 2017). Também existem evidências de desigualdades no acesso a muitos tratamentos curativos contra o câncer relacionadas à idade, onde os idosos seriam menos propensos à cirurgia e quimioterapia, por exemplo, priorizando a manutenção da 
qualidade de vida. Nas leucemias, o prognóstico de pacientes idosos em idades avançadas é reservado e a recomendação de regime de indução de dose padrão ou de baixa intensidade permanece controversa (CANCER RESEARCH UK, 2019; Yi C, et al., 2016).

Apesar das limitações nos dados obtidos no SIH segundo os procedimentos realizados nas internações hospitalares entre 2008 e 2016, a sua caracterização revelou que $68,2 \%$ dos atendimentos foram realizados em caráter de urgência e, destes, quase 15\% morreram. Para as leucemias, esse valor correspondeu a 72,4\% dos atendimentos, sendo que o código "internação para quimioterapia de leucemias agudas/crônicas agudizadas" foi o mais frequente $(60,1 \%)$, corroborando o diagnóstico tardio desses pacientes.

O diagnóstico precoce aumenta a sobrevida de pacientes com câncer, do mesmo modo que aumenta as possibilidades de tratamento. Ainda assim, muitos pacientes ainda são diagnosticados em atendimentos de emergência, o que aumenta a probabilidade de essas doenças estarem em estádios avançados contribuindo para redução da sobrevida e pior experiência no tratamento do câncer (CANCER RESEARCH UK, 2019).

De 2008 a 2016, foram realizados 17.394 TCTH, autorizados pelo SUS. Destes, 51,4\% foram do tipo autogênico, uma das estratégias terapêuticas aplicadas ao mieloma múltiplo, apesar de ser uma doença ainda sem tratamento curativo.

Nesses casos, o TCTH autólogo ajuda na melhora da resposta ao tratamento e prolonga a sobrevida dos pacientes, embora grande parte apresente recaída durante a evolução da doença. O diagnóstico tardio e, consequentemente, do tratamento, está associado a um impacto significante no decurso clínico do mieloma múltiplo, corroborando os achados de tendência crescente nas mortes pela doença entre os idosos (SALEMA CLZ e CARVALHO C, 2019). Em estudo observacional realizado em 23 centros da América Latina entre 2007 e 2009, com um total de 852 pacientes com mieloma, foi observada prevalência do sexo masculino (aproximadamente 53\%) e no grupo de pacientes não elegíveis para transplante, a média de idade foi de 67,4 anos. Já entre os pacientes elegíveis para transplante, a idade média foi de 54,7 anos (HUNGRIA VT, et al., 2017).

A tendência de incidência do mieloma em países como a Suíça, entre 1994 e 2013, mostrou-se crescente com o tempo devido a alterações demográficas, mas nos Estados Unidos, essas taxas não apresentaram mudanças significativas nos últimos 10 anos. Quanto à sobrevida relativa em 5 e 10 anos, houve um aumento substancial nesses países também associado aos avanços no tratamento da doença, sendo que a mortalidade entre os americanos apresentou curva decrescente, com redução de 0,7\% ao ano entre 2005 e 2014 (HOWLADER et al., 2017; ANDRES M, et al., 2018).

Essa pesquisa evidenciou diferenças importantes entre as regiões brasileiras. As regiões Sul e Sudeste do país concentraram quase $70 \%$ das mortes e das internações por doenças oncohematológicas, consequente também à maior concentração de serviços médicos e leitos hospitalares nos Estados mais desenvolvidas do país. Ademais, os maiores incrementos anuais dessas taxas foram observados no Sul e Nordeste, destacando a necessidade comum de centros oncológicos especializados para otimizar a assistência, principalmente em regiões onde as taxas de mortalidade mostram crescimento.

Os estudos epidemiológicos sobre doenças oncohematológicas em adultos são escassos na literatura brasileira, ainda mais nos últimos cinco anos, o que dificultou a discussão desses achados na busca de explicações razoáveis para as diferenças marcantes entre as regiões. A heterogeneidade geográfica da mortalidade por câncer no Brasil pode estar associada a exposições a fatores de risco específicos para essa doença, além de ser influenciada por fatores temporais, socioeconômicos, políticos e, como foi discutido anteriormente, pela oportunidade de acesso ao diagnóstico e tratamento de câncer (WUNSCH VF e MONCAU JE, 2002; BOCCOLINI PMM, et al., 2015; MARTINS SM, 2020).

Boccolini PMM, et al. (2015) analisaram a tendência de mortalidade por linfoma não Hodgkin em adultos no Brasil, entre 1980 e 2012. Foi observado aumento significativo das taxas de mortalidade nas regiões, porém a região Sudeste foi a única que apresentou tendência de diminuição das taxas de mortalidade por LNH, estatisticamente significante, no período de 1999 a 2012. As taxas também foram maiores na faixa etária de 60 anos ou mais e entre os homens, com destaque para os fatores de risco já conhecidos da doença 
enquanto fatores explicativos, incluindo a discussão sobre a exposição aos agrotóxicos, amplamente utilizados no Brasil, em concordância com outro estudo na área (COSTA VIB, et al., 2017).

As melhorias implementadas no sistema de notificação de mortes, com redução das causas mal-definidas, ao longo do período de estudo podem também explicar o importante incremento nas taxas de mortalidade nas regiões Norte e Nordeste. Outros estudos destacam a escassez de recursos financeiros e investimentos no diagnóstico dessas doenças como fatores associados à essa subnotificação, principalmente na região Norte (SILVA-JUNIOR AL, et al., 2019).

Sobre esse aspecto, deve-se considerar ainda a correlação positiva entre mortalidade por câncer e condições socioeconômicas, expressadas pelos indicadores de renda e esperança de vida, representadas através das diferenças nas taxas de óbito por câncer observadas entre as regiões brasileiras neste e em outros estudos.

Desse modo, as populações do Sul e Sudeste são mais privilegiadas e têm maior acesso aos recursos diagnósticos e terapêuticos, ou seja, possuem serviços de vigilância epidemiológica do câncer estruturados nessas áreas, o que reflete na qualidade nos registros de óbito (RIBEIRO IB, et al., 2016). Nesse sentido, o presente estudo tem como limitação a utilização de dados secundários, sujeitos à falhas de registro e para morbidade hospitalar, refletem apenas as internações financiadas pelo SUS.

\section{CONCLUSÃO}

Poucas investigações abordam a tendência de morbimortalidade por doenças oncohematológicas em adultos no Brasil. Observou-se tendência crescente nessas taxas entre 2002 e 2016, com incremento anual de 0.04 óbitos/ano por Leucemias, de 0.03/ano por Linfomas e de 0.04/ano por Mieloma, maior número de mortes entre os homens e hospitalizações nos idosos, com maior proporção de atendimentos em caráter de urgência. Sul e Sudeste concentraram quase $70 \%$ das mortes e das internações, porém houve um incremento importante nessas taxas no Nordeste. A análise desses dados permitiu ampliar o conhecimento sobre o perfil epidemiológico, bem como compreender o impacto dos tratamentos e a qualidade dos sistemas de saúde, produzindo indicadores de gestão em Saúde Pública.

\section{REFERÊNCIAS}

1. ANDRES M, et al. Trends of incidence, mortality, and survival of multiple myeloma in Switzerland between 1994 and 2013. Cancer Epidemiol. 2018; 53:105-110.

2. BRASIL. Ministério da Saúde. Secretaria de Atenção a Saúde. Portaria nº2439/GM de 08/12/2005. Política Nacional de Atenção Oncológica. Brasil: Ministério da Saúde, 2005.

3. BRASIL. Tribunal de Contas da União. Secretaria de Fiscalização e Avaliação de Programas de Governo. Relatório de Auditoria Operacional: Política Nacional de Atenção Oncológica. Brasília: TCU, 2011.132 p.

4. BRASIL. Ministério da Saúde. Secretaria de Atenção a Saúde. Portaria n 1399, de 17 de dezembro de 2019. Redefine os critérios e parâmetros referenciais para a habilitação de estabelecimentos de saúde na alta complexidade em oncologia no âmbito do SUS. Brasil: Ministério da Saúde, 2019.

5. BOCCOLINI PMM, et al. Tendência de mortalidade por linfomas não Hodgkin no Brasil, 1980 a 2012. Cad. Saúde Colet. 2015; 23(2): 188-197.

6. CANCER RESEARCH UK. Cancer in the UK. Cancer Research UK: London, 2019. 24 p.

7. CANCER RESEARCH UK. 2020 In: Statistics by cancer type. Disponível em: https://www.cancerresearchuk.org/health-professional/cancer-statistics/statistics-by-cancer-type. Acesso em: 08 abr. 2020.

8. COSTA VIB, et al. Exposição ambiental e ocupacional a agrotóxicos e o linfoma não Hodgkin. Saúde debate. 2017; 41(112): 49-62.

9. FERLAY J, et al. Global and Regional Estimates of the Incidence and Mortality for 38 Cancers: GLOBOCAN 2018. Lyon: International Agency for Research on Cancer/World Health Organization; 2018.

10. GRANJEIRO CF, et al. Epidemiological Profile of Patients with Hematological Neoplasms in an Oncological Hospital of Mato Grosso. J Health Sci. 2018; 20(4): 232-237.

11. GUERRA MR, et al. Magnitude e variação da carga da mortalidade por câncer no Brasil e Unidades da Federação, 1990 e 2015. Rev bras epidemiol. 2017; 20(1): 102-115.

12. HAGSTRÖM H, et al. Body composition measurements and risk of hematological malignancies: A population-based cohort study during 20 years of follow-up. PLoS One. 2018; 13(8): e0202651. 
13. HOWLADER N, et al. SEER Cancer Statistics Review, 1975-2014. National Cancer Institute, 2017.

14. HOWLADER N, et al. SEER Cancer Statistics Review, 1975-2017. National Cancer Institute, 2020.

15. HUNGRIA VTM, et al. Observational study of multiple myeloma in Latin America. Ann Hematol. 2017; 96(1): 65-72.

16. INSTITUTO NACIONAL DE CÂNCER. In: Tipos de câncer. Rio de Janeiro: INCA, 2018. Disponível em: https://www.inca.gov.br/tipos-de-cancer/leucemia/profissional-de-saude. Acesso em: 25 maio 2020.

17. INSTITUTO NACIONAL DE CÂNCER. Estimativa 2020: incidência de câncer no Brasil. Rio de Janeiro: INCA, 2019; $120 \mathrm{p}$.

18. MARTINS SM. Morte por Câncer e Doença Cardiovascular entre Dois Brasis. Arq. Bras. Cardiol. 2020; $114(2)$ : 207 208.

19. OLIVEIRA MD, et al. Avaliação do perfil epidemiológico das neoplasias onco-hematológicas de pacientes atendidos pelo instituto de câncer de três lagoas, no período de 2014 a 2018. Braz. J. of Develop. 2020; 6(2): 7301-7314.

20. OMS. Organização Mundial da Saúde. 2020. In: Global Health Expenditure Database. Disponível em: http://apps.who.int/nha/database/ViewData/Indicators/en. Acesso em: 08 abr. 2020.

21. RIBEIRO IB, et al. Desigualdades socioeconômicas e mortalidade por câncer: um estudo ecológico no Brasil. Revista Brasileira em Promoção da Saúde. 2016; 29(3): 350-356.

22. SALDIVA PHN, VERAS M. Gastos públicos com saúde: breve histórico, situação atual e perspectivas futuras. Estud. av. 2018; 32(92): 47-61.

23. SALEMA CLZ, CARVALHO C. Diagnósticos, tratamentos e prognósticos do mieloma múltiplo. Rev Ciên Saúde. 2019; 4(1): 1-9.

24. SANT M, et al. Survival for haematological malignancies in Europe between 1997 and 2008 by region and age: results of EUROCARE-5, a population-based study. The Lancet Oncology. 2015; 15(9): 931-942.

25. SILVA FS, LATORRE MRDS. Sobrevida das leucemias linfoides agudas em crianças no município de São Paulo, Brasil. Cad. Saúde Pública. 2020; 36(3): 1-9.

26. SILVA-JUNIOR AL, et al. Acute lymphoid and myeloid leukemia in a Brazilian Amazon population: Epidemiology and predictors of comorbidity and deaths. PLoS One. 2019; 14(8): e0221518.

27. WÜNSCH VF, MONCAU JE. Mortalidade por câncer no Brasil 1980-1995: padrões regionais e tendências temporais. Rev. Assoc. Med. Bras. 2002; 48(3): 250-257.

28. $\mathrm{YI} \mathrm{C}$, et al. The outcome and prognostic factors of 248 elderly patients with acute myeloid leukemia treated with standard-dose or low-intensity induction therapy. Medicine (Baltimore). 2016; 95(30): e4182.

29. YE X, et al. Long-term time trends in incidence, survival and mortality of lymphomas by subtype among adults in Manitoba, Canada: a population-based study using cancer registry data. BMJ open. 2017; 7(7): e015106. 